\title{
The potential role of oxytocin and perinatal factors in the pathogenesis of autism spectrum disorders - review of the literature
}

\author{
Melinda Vanya ${ }^{\mathrm{a}, \mathrm{b}, *}$, Szabina Szucs $^{\mathrm{c}}$, Agnes Vetro $^{\mathrm{c}}$, Gyorgy Bartfai $^{\mathrm{a}}$ \\ a Department of Obstetrics and Gynaecology, Albert Szent-Gyorgyi Clinical Centre, University of Szeged, Szeged, Hungary \\ ${ }^{\mathrm{b}}$ KF TFK Health Research and Health Promotion Research Group, Kecskemet, Hungary \\ ${ }^{\mathrm{c}}$ Division of Adolescent Psychiatry, Department of Paediatrics, Albert Szent-Gyorgyi Clinical Centre, University of Szeged, Szeged, Hungary
}

\section{A R T I C L E I N F O}

\section{Keywords:}

Autism spectrum disorder, behavior

Gene

Cesarean section, oxytocin, oxytocin-receptor polymorphism

\begin{abstract}
A B S T R A C T
Autism Spectrum Disorders (ASD) are characterized by: social and communication impairments, and by restricted repetitive behaviors. The aim of the present paper is to review abnormalities of oxytocin (OXT) and related congenital malformations in ASD. A literature search was conducted in the PubMed database up to 2016 for articles related to the pathomechanism of ASD, abnormalities of OXT and the OXT polymorphism in ASD. The pathomechanism of ASD has yet to be. The development of ASD is suggested to be related to abnormalities of the oxytocin-arginin-vasopressin system. Previous results suggest that OXT and arginine vasopressin (AVP) may play a role in the etiopathogenesis of ASD.
\end{abstract}

\section{Introduction}

Autism spectrum disorder was previously perceived as a rare disorder, but increasing recognition, understanding, and awareness is changing this perception, and it is now considered to be a relatively common condition. Prevalence rate estimates vary between $1 \%$ and $2 \%$ in school age children (Ben-Ari, 2015).

ASD has a great impact on the quality of life in the affected children and their family. ASD seems to be a multifactorial disease with the interaction of genetic and environmental factors. Evidence supporting the hypothesis that perinatal outcome can play a role in the etiology of is ASD increasing. The present paper focuses on recent publications which have demonstrated the role of oxytocin, and prenatal and perinatal factors associated with an increased risk of ASD (Fakhoury, 2015; Muotri et al., 2016). In this paper, several methodological advantages have helped to support and extend this hypothesis.

\section{Methods}

\subsection{Search strategy}

Published data were used in our paper; therefore, no ethical approval was required.

The authors searched for the terms 'ASD' OR 'perinatal outcome' OR 'oxytocin' in the following databases: Medline, Pubmed, Scopus, Index Medicus, and Science Direct.
The following outcome measures were analyzed (1) oxytocin and oxytocin receptor defect in the development of ASD; (2) the relative risk of perinatal factors and congenital defects.

- Qualitative data: after screening the title and abstract, all texts not presenting data on oxytocin-receptor related to ASD were discarded.

- Quantitative data: only papers presenting original work with analysis on the relationship between oxytocin; cesarean section, perinatal outcome, and development of ASD were included. Reference lists from these papers were read to search for other possible relevant papers. Abstracts of scientific meetings, review papers, anecdotal case reports, duplicate papers, and editorials were excluded.

\section{Results}

\subsection{The role of oxytocin}

Social and communication difficulties are the most characteristic disturbances in ASD, and they are two of the core symptom domains. Repetitive behavior, and restricted activities and interests constitute the third domain of symptoms, as listed in the DSM-IV (American Psychiatric Association, 2000).

Oxytocin (OXT) is a nine-amino-acid peptide that is synthesized in the paraventricular and supraoptic nucleus of the hypothalamus and released into the bloodstream by axon terminals in the posterior pituitary (Lee et al., 2009). The best known effects in women of

\footnotetext{
* Correspondence to: Department of Obstetrics and Gynaecology, Albert Szent-Gyorgyi Clinical Centre, University of Szeged, 1 Semmelweis Street, H-6725 Szeged, Hungary.

E-mail address: vmelinda74@gmail.com (M. Vanya).
} 
reproductive age are in stimulating uterine contractions during labor and in regulating lactation. Furthermore, it is important to emphasize that OXT and the structurally similar peptide AVP are directly released within the brain, where they play a key role in regulating social affiliative behaviors, including sexual behavior, and social recognition (Lee et al., 2009; Harony, 2010; Caldwell, 2008). Besides these functions, the genes coding for the production of OXT and AVP are located on the same chromosome, 20p13, while the genes for oxytocin receptor (OXTR) are located on chromosome 3. OXT plays a role in increasing trust, empathy, eye contact, face memory, and social interaction (Donaldson and Young, 2008). AVP, which is well known for its antidiuretic effect, influences social behavior as well (Harony and Wagner, 2010). Repetitive behaviors are possibly influenced by OXT and AVP since they act as neuromodulators, targeting the hippocampus, amygdala, striatum, hypothalamus, and nucleus accumbens (Insel, 2010; Israel, 2008; Green, 2010). Lastly, it is through their neuromodulatory role that OXT and AVP are implicated in other behaviors such as stress-response, learning, and memory (Donaldson ZR, 2008). Overall, they play a critical role in regulating social behavior from the early phases of development, and thus are likely to be implicated in ASD unfolding. For more than a decade, several authors and teams have assessed the possibility that OXT may be influenced in the social symptoms of ASD (Insel et al., 2010; Green et al., 2010; Israel et al., 2008). Abnormalities in OXT/AVP in ASD might be due to different mechanisms, such as a reduction in hormonal levels as a result of diminished synthesis, an alteration in hormonal release, or insufficient amounts of these hormones as neuromodulators in the brain possibly because of receptor abnormalities (Israel et al., 2008; Gregory et al., 2009). Furthermore, it has been suggested that an alteration in hormonal release might be secondary to an accelerated synaptic pruning, and/or alteration of the glia (Wang and Hatton, 2009). Several neuronal network, neurotransmission and hormone systems are probably involved in ASD. Currently, neural systems of the amygdala, the mesocorticolimbic dopamine system and the OXT system are among the most important ones (Neuhaus et al., 2010). The relevance of OXT as implicated in the facilitation of social information processing is possibly obtained through auditory and visual pathways. Finally, OXT is involved in regulating stress and fear reactivity, possibly promoting social interaction in individuals with ASD that often experience anxiety associated with nuclear disturbances in social interaction (Bartz et al., 2008).

\subsection{Genetic studies}

Genetic studies also suggested a role of OXT in ASD. The region of chromosome 3 , in which OXTR is located is now considered a susceptibility locus, as it has repeatedly been found to be implicated in ASD (Liu et al., 2016; Jansen et al., 2006; Ebstein et al., 2009; Andari et al., 2010; Green et al., 2001; Guastella et al., 2010; Wang and Hatton, 2009; Sala et al., 2011; Schmidt et al., 2012; Guastella et al., 2008; Kurth et al., 2011). Wu et al. have reported an association between two single nucleotide polymorphisms (SNP) in the OXTR related to the occurrence of ASD (Wu et al., 2005). Jacob et al. have also found an association between OXTR and autism; specifically, a significant association was detected at rs2254298, but in contrast with Wu et al., not at rs53576 (Jacob, 2007).

Similarly, Lerer et al. have shown that SNPs and haplotypes in the OXTR gene confer risk for ASD, and in addition, they have observed an association with IQ and adaptive functions measured with total Vineland Adaptive Behaviors Scales (communication, daily living skills, and socialization subdomains) (Lerer et al., 2008). Recently, a study with a large cohort of 2333 individuals with ASD has demonstrated an association between three markers implicated in ASD susceptibility, rs2268493, rs1042778, and rs7632287, further strengthening the hypothesis that functional polymorphisms of OXTR may contribute to ASD risk (Campbell et al., 2011). Similar conclusions have been drawn in another study by Wertmer et al. observing a significant genetic variation in the OXTR gene in patients with ASD including atypical and high-functioning individuals (Wermter et al., 2010). These findings point to an epigenetic dysregulation of OXTR. How this process might unfold is currently unknown, but it is important to note that if further evidence is discovered, it might explain the failure to identify sequence alterations in many candidate genes of ASD, e.g., an epigenetic alteration would be implicated in ASD phenotype expression (Gurrieri et al., 2009).

\subsection{Perinatal factors}

ASD is influenced by a variety of genetic, environmental, and possibly immunological factors that act during critical periods to alter key developmental processes. Some pharmaceutical drugs, toxicants, and metabolic and nutritional factors have been identified in epidemiological studies to increase the risk for autism, especially during the prenatal period. Immunological risk factors including maternal infection during pregnancy, autoantibodies to fetal brain proteins, and familial autoimmune diseases have consistently been observed in multiple studies, as well as immune abnormalities in individuals with ASD.

Etiological hypotheses suggest a strong genetic component and a possible interaction between the epigenetic factors in early fetal development (Kolevzon et al., 2007).

The suggested environmental factors include nutritional and immune function related risk factors such as lack of vitamin D and folic acid supplementation, and metabolic syndrome (Gialloreti et al., 2014). It can be concluded that the rate of cesarean sections was also significantly higher in the case group than in the control group (Kolevzon et al., 2007; Gialloreti et al., 2014; Curran et al., 2015). Shieve et al. have concluded that three perinatal risk factors (preterm birth, small for gestational age, and cesarean section) notably contribute to ASD risk (Shieve et al., 2014). Nilsen et al. (2013) analyzed clinical data on a large cohort of pregnant women in 2014 concerning the perinatal outcomes and ASD. The results suggest that cesarean section, prenatal folic acid use, maternal smoking in the prenatal period, low birth weight and preterm birth have increased the risk of developing ASD.

\subsection{Congenital anomalies}

Earlier population-based register studies on the association between ASD and syndromes are scanty, as the syndromes are rare (Lee et al., 2015).

Kielinen et al. have reported a population based study of 187 ASD children with one Klinefelter syndrome (47, XXY), (0.5\%) one 47/XYY (0.5\%, one Down syndrome (0.5\%), four Fragile X syndromes $(2.1 \%)$ two fetal alcoholic syndrome (1.1\%), and one Sotos syndrome $(0.5 \%$, one tuberous sclerosis (0.5\%) (Kielinen et al., 2004).

\section{Discussion}

Considering the complex characteristics of ASD, which are a combination of biologic heterogeneity and phenotypic heterogeneity, it is difficult to choose and develop an effective treatment. Since oxytocin is responsible for not only maternal behavior but also core symptom domains of ASD, such as social interaction and repetitive behaviors, association with the pathogenesis of ASD is highly possible. There are various ongoing animal, human, and clinical studies assessing the potential disruption of oxytocin function or secretion leading to the etiology of ASD. SNP of the OXTR is the first step in the recognition of new treatment molecules. Through clinical trials applying oxytocin in patients with ASD, future clinical applications are promising. Therefore, further research should be carried out to develop treatment options for children with ASD (Ben-Ari, 2015; Curran et al., 
2015; Egawa et al., 2013).

\section{Funding statement}

N.a.

\section{Conflict of interest}

The authors have no conflict of interest.

\section{Acknowledgement}

No.

\section{References}

American Psychiatric Association, 2000. Diagnostic and statistical manual of mental disorders. 4th ed. Text revision Washington.

Andari, E., Duhamel, J.R., Zalla, T., Herbrecht, E., Leboyer, M., Sirigu, A., 2010. Promoting social behavior with oxytocin in high-functioning autism spectrum disorders. Proc. Natl. Acad. Sci. USA. 107 (9), 4389-4394.

Ben-Ari, Y., 2015. Is birth a critical period in the pathogenesis of autism spectrum disorders? Nat. Rev. Neurosci. 16 (8), 498-505.

Caldwell, H.K., Lee, H.J., Macbeth, A.H., Young, W.S., 2008. Vasopressin: behavioral roles of an "original" neuropeptide. Prog. Neurobiol. 84 (1), 1-24.

Campbell, D.B., Datta, D., Jone, s.S.T., Batey, Lee E., Sutcliffe, J.S., Hammock, E.A., Levitt, P., 2011. Association of oxytocin receptor (OXTR) gene variants with multiple phenotype domains of autism spectrum disorders. J. Neurodev. Disord. 3 (2), $101-112$.

Curran, E.A., O'Neill, S.M., Cryan, J.F., Kenny, L.C., Dinan, T.G., Khashan, A.S., Kearney, P.M., 2015. Research Review: birth by caesarean section and development of autism spectrum disorder and attention-deficit/hyperactivity disorder: a systematic Review and meta-analysis. J. Child. Psychol. Psychiatry 56 (5), 500-508.

Donaldson, Z.R., Young, L.J., 2008. Oxytocin, vasopressin, and the neurogenetics of sociality. Science 322 (5903), 900-904.

Ebstein, R.P., Israel, S., Lerer, E., Uzefovsky, F., Shalev, I., Gritsenko, I., et al., 2009. Arginine vasopressin and oxytocin modulate human social behavior. Ann. New Y. Acad. Sci. 1167, 87.

Egawa, J., Watanabe, Y., Endo, T., Tamura, R., Masuzawa, N., Someya, T., 2013. Association between OXTR and clinical phenotypes of autism spectrum disorders. Psychiatry Res. 208 (1), 99-100.

Fakhoury, M., 2015. Autistic spectrum disorders: a review of clinical features, theories and diagnosis. Int. J. Dev. Neurosci. 43, 70-77.

Green, J.J., Hollander, E., 2010. Autism and oxytocin: new developments in translational approaches to therapeutics. Neurotherapeutics 3, 250-257.

Green, L., Fein, D., Modahl, C., Feinstein, C., Waterhouse, L., Morris, M., 2001. Oxytocin and autistic disorder: alterations in peptide forms. Biol. Psychiatry 50, 609-613.

Gregory, S.G., Connelly, J.J., Towers, A.J., Johnson, J., Biscocho, D., Markunas, C.A., Lintas, C., et al., 2009. Genomic and epigenetic evidence for oxytocin receptor deficiency in autism. BMC Med. 7, 62.

Gialloreti, L.E., Benvenuto, A., Benassi, F., Curatolo, P., 2014. Are caesarean sections, induced labor and oxytocin regulation linked to autism spectrum disorders? Med. Hypotheses 82 (6), 713-718.

Guastella, A.J., Mitchell, P.B., Dadds, M.R., 2008. Oxytocin increases gaze to the eyeregion of human faces. Biol. Psychiatry 63 (1), 3-5.

Guastella, A.J., Einfeld, S.L., Gray, K.M., Rinehart, N.J., Tonge, B.J., Lambert, T.J., et al., 2010. Intranasal oxytocin improves emotion recognition for youth with autism spectrum disorders. Biol. Psychiatry 67, 692-694.
Gurrieri, F., Neri, G., 2009. Defective oxytocin function: a clue to understanding the cause of autism? BMC Med. 7, 63.

Harony, H., Wagner, S., 2010. The contribution of oxytocin and vasopressin to mammalian social behavior: potential role in autism spectrum disorder. Neurosignals 18, 82-97.

Insel, T.R., 2010. The challenge of translation in social neuroscience: a review of oxytocin, vasopressin, and affiliative behavior. Neuron 65, 768-779.

Israel, S., Lerer, E., Shalev, I., Uzefovsky, F., Reibold, M., Bachner-Melman, R., et al., 2008. Molecular genetic studies of the arginine vasopressin 1a receptor (AVPR1a) and the oxytocin receptor (OXTR) in human behaviour: from autism to altruism with some notes in between. Prog. Brain Res. 170, 435-449.

Jacob, S., Brune, C.W., Carter, C.S., Leventhal, B.L., Lord, C., Cook, E.H., Jr., 2007. Association of the oxytocin receptor gene (OXTR) in Caucasian children and adolescents with autism. Neurosci. Lett. 417, 6-9.

Jansen, L.M., Gispen-de Wied, C.C., Wiegant, V.M., Westenberg, H.G., Lahuis, B.E., van Engeland, H., 2006. Autonomic and neuroendocrine responses to a psychosocial stressor in adults with autistic spectrum disorder. J. Autism Dev. Disord. 36, 891-899.

Kielinen, M., Rantala, H., Timonen, E., Linna, S.L., Moilanen, I., 2004. Associated medical disorders and disabilities in children with autistic disorder: a populationbased study. Autism 8 (1), 49-60.

Kolevzon, A., Gross, R., Reichenberg, A., 2007. Prenatal and perinatal risk factors for autism: a review and integration of findings. Arch. Pediatr. Adolesc. Med. 161 (4), 326-333.

Kurth, F., Narr, K.L., Woods, R.P., O'Neill, J., Alger, J.R., Caplan, R., et al., 2011. Diminished gray matter within the hypothalamus in autism disorder: a potential link to hormonal efects? Biol. Psychiatry 70, 278-282.

Lee, H.J., Macbeth, A.H., Pagani, J.H., Young, W.S., 2009. Oxytocin: the great facilitator of life. Prog. Neurobiol. 88, 127-151.

Lee, S.Y., Lee, A.R., Hwangbo, R., Han, J., Hong, M., Bahn, G.H., 2015. Is Oxytocin application for autism spectrum disorder Evidence-based? Exp. Neurobiol. 24 (4), 312-324.

Lerer, E., Levi, S., Salomon, S., Darvasi, A., Yirmiya, N., Ebstein, R.P., 2008. Association between the oxytocin receptor (OXTR) gene and autism: relationship to Vineland Adaptive Behavior Scales and cognition. Mol. Psychiatry 13, 980-988.

Liu, X., Kawashima, M., Miyagawa, T., Otowa, T., Latt, K.Z., Thiri, M., et al., 2016. Novel rare variations of the oxytocin receptor gene in autism spectrum disorder individuals. Hum. Genome Var. 14 (3), 15046.

Muotri, A.R., 2016. The human model: changing focus on autism Research. Biol. Psychiatry 79, 642-649.

Neuhaus, E., Beauchaine, T.P., Bernier, R., 2010. Neurobiological correlates of social functioning in autism. Clin. Psychol. Rev. 30, 733-748.

Nilsen, R.M., Surén, P., Gunnes, N., Alsaker, E.R., Bresnahan, M., Hirtz, D., et al., 2013. Analysis of self-selection bias in a population-based cohort study of autism spectrum disorders. Paediatr. Perinat. Epidemiol. 27 (6), 553-563.

Sala, M., Braida, D., Lentini, D., Busnelli, M., Bulgheroni, E., Capurro, V., et al., 2011. Rescue of impaired cognitive flexibility social deficits increased aggression and seizure susceptibility in oxytocin receptor null mice: a neurobehavioral model of autism. Biol. Psychiatry 69, 875-882.

Schmidt, R.J., Tancredi, D.J., Ozonoff, S., Hansen, R.L., Hartiala, J., Allayee, H., et al., 2012. Maternal periconceptional folic acid intake and risk of autism spectrum disorders and developmental delay in the CHARGE (CHildhood autism Risks from Genetics and Environment) case-control study. Am. J. Clin. Nutr. 96 (1), 80-89.

Wang, Y.F., Hatton, G.I., 2009. Astrocytic plasticity and patterned oxytocin neuronal activity: Dynamic interactions. J. Neurosci. 29, 1743-1754.

Wermter, A.K., Kamp-Becker, I., Hesse, P., Schulte-Körne, G., Strauch, K., Remschmidt, H., et al., 2010. Evidence for the involvement of genetic variation in the oxytocin receptor gene (OXTR) in the etiology of autistic disorders on high-functioning level. Am. J. Med. Genet. B. Neuropsychiatr. Gene. t 153B, 629-639.

Wu, S., Jia, M., Ruan, Y., Liu, J., Guo, Y., Shuang, M., et al., 2005. Positive association of the oxytocin receptor gene (OXTR) with autism in the Chinese Han population. Biol. Psychiatry 58 (1), 74-77. 